Article

\title{
Geometric Interpretation of Errors in Multi-Parametrical Fitting Methods Based on Non-Euclidean Norms
}

\author{
George Livadiotis
}

Southwest Research Institute, Space Science \& Engineering, San Antonio, TX 78238, USA; glivadiotis@swri.edu; Tel.: +1-210-274-4028

Received: 29 September 2019; Accepted: 25 October 2019; Published: 29 October 2019

\begin{abstract}
The paper completes the multi-parametrical fitting methods, which are based on metrics induced by the non-Euclidean $L_{q}$-norms, by deriving the errors of the optimal parameter values. This was achieved using the geometric representation of the residuals sum expanded near its minimum, and the geometric interpretation of the errors. Typical fitting methods are mostly developed based on Euclidean norms, leading to the traditional least-square method. On the other hand, the theory of general fitting methods based on non-Euclidean norms is still under development; the normal equations provide implicitly the optimal values of the fitting parameters, while this paper completes the puzzle by improving understanding the derivations and geometric meaning of the optimal errors.
\end{abstract}

Keywords: fitting; non-Euclidean norm; fitting errors; optimization

\section{Introduction}

The keys to evaluating an experimental result-e.g., compare it with the result anticipated by theories-require first the right selection of potential statistical tools and techniques for correctly processing and analyzing this result. This "processing and analyzing" involves two general types of approximation problems: One problem concerns a function fitting to given set of data. The other problem arises when a function is given analytically by an explicit mathematical type but we would like to find an alternative function with simpler form.

Let $V\left(x ; p_{1}, p_{2}, \ldots, p_{n}\right)$, with $x \in D \subseteq \mathfrak{R}$ and $\left(p_{1}, p_{2}, \ldots, p_{n}\right) \in\left\{D_{p_{1}} \otimes D_{p_{2}} \otimes \ldots \otimes D_{p_{n}}\right\} \subseteq \mathfrak{R}^{n}$, denote a multi-parametrical approximating function [1-6], symbolized as $V\left(x ;\left\{p_{k}\right\}\right)$, for short.

The widely used, traditional fitting method of least squares involves minimizing the sum of the squares of the residuals, i.e., the squares of the differences between the function $f(x)$ and the approximating function that represents the statistical model, $V(x)$. However, the least-square method is not unique. For instance, the absolute deviations minimization can also be applied. Generally, as soon as the desired norm of the metric space is given, the respective method of deviations minimization is defined. The least-square method is based on the Euclidean norm, while the alternative absolute deviations method is based on the uniform or Taxicab norm. In general, an infinite number of fitting methods can be defined, based on the metric space induced by the $L_{q}$-norm; this case is studied here in detail.

Given the metric induced by the $L_{q}$-norm, the functional of the total $L_{q}$-normed residuals [7-12], noted also as total deviations (TD), between the fixed $f(x)$ and the approximating $V\left(x ;\left\{p_{k}\right\}\right)$ functions in the domain $D$, is given by:

$$
T D_{q}\left(\left\{p_{k}\right\}\right)^{q}=\int_{x \in D}\left|V\left(x ;\left\{p_{k}\right\}\right)-f(x)\right|^{q} d x .
$$


The functional of total deviations, $T D_{q}\left(\left\{p_{k}\right\}\right)^{q}$, is expanded (Taylor series) near its local minimum:

$$
T D_{q}\left(\left\{p_{k}\right\}\right)^{q}=A_{0}(q)+\sum_{k_{1}, k_{2}=1}^{n} A_{2, k_{1} k_{2}}(q) \cdot\left(p_{k_{1}}-p_{k_{1}}{ }^{*}\right) \cdot\left(p_{k_{2}}-p_{k_{2}}{ }^{*}\right)+O\left(\left|p_{k}-p_{k}{ }^{*}\right|^{3}\right)
$$

where

$$
A_{0}(q)=T D_{q}\left(\left\{p_{k}^{*}\right\}\right)^{q},
$$

is the total deviation function at its global minimum, while

$$
A_{2, k_{1} k_{2}}(q)=\left.\frac{1}{2} \cdot \frac{\partial^{2}}{\partial p_{k_{1}} \partial p_{k_{2}}} T D_{q}\left(\left\{p_{k}\right\}\right)^{q}\right|_{\left\{p_{k}\right\}=\left\{p_{k}^{*}\right\}},
$$

is the Hessian matrix at this minimum, where all the components are positive, i.e., $A_{0}, A_{2, k_{1} k_{2}} \geq 0$, $\forall k_{1}, k_{2}=1,2, \ldots, n$.

By expanding the approximating function $V\left(x ;\left\{p_{k}\right\}\right)$ near the TD's minimum, [7] showed the following equations:

$$
A_{0}(q)=\int_{x \in D}|u|^{q} d x
$$

and

$$
\begin{aligned}
& A_{2, k_{1} k_{2}}(q)=\delta_{1 q} \cdot \gamma_{k_{1} k_{2}}+ \\
& \frac{q}{2} \cdot \int_{x \in D}\left[|u|^{q-1} \cdot \operatorname{sgn}(u) \cdot \frac{\partial^{2} V\left(x ;\left\{p_{k}^{*}\right\}\right)}{\partial p_{k_{1}} \partial p_{k_{2}}}+(q-1) \cdot|u|^{q-2} \cdot \frac{\partial V\left(x ;\left\{p_{k}^{*}\right\}\right)}{\partial p_{k_{1}}} \cdot \frac{\partial V\left(x ;\left\{p_{k}{ }^{*}\right\}\right)}{\partial p_{k_{2}}}\right] d x,
\end{aligned}
$$

where

$$
\gamma_{k_{1} k_{2}} \equiv \sum_{\substack{\forall i: \\ u\left(x_{i}\right)=0}} \frac{1}{\left|u^{\prime}\left(x_{i}\right)\right|} \cdot \frac{\partial V\left(x_{i} ;\left\{p_{k}^{*}\right\}\right)}{\partial p_{k_{1}}} \cdot \frac{\partial V\left(x_{i} ;\left\{p_{k}^{*}\right\}\right)}{\partial p_{k_{2}}}
$$

The normal equations are given by

$$
\int_{x \in D}|u|^{q-1} \cdot \operatorname{sgn}(u) \cdot \frac{\partial V\left(x ;\left\{p_{k}^{*}\right\}\right)}{\partial p_{l}} d x=0, \forall l=1,2, \ldots, n .
$$

where we set $u=u(x) \equiv V\left(x ;\left\{p_{k}^{*}\right\}\right)-f(x)$ for short.

The purpose of this paper is to present the geometric interpretation of the errors of the optimal parameter values, derived from a multi-parametrical fitting, based on a metric induced by the non-Euclidean $L_{q}$-norm. In Section 2, we derive the smallest possible value of the variation of the total deviations from its minimum, $\delta T D$, also called, the error of the total deviations value. In Section 3 , we describe the geometric interpretation of the errors of the optimal parameter values, while in Section 4, we use this geometry to derive the exact equations that provide these errors. In Section 5 , we apply the developed formulation for the 1-dim and 2-dim cases. Finally, Section 6 summarizes the conclusions.

\section{The Error of the Total Deviation Values}

The total deviations functional, $T D_{a}\left(\left\{p_{k}\right\}\right)^{q}$, has a minimum value $A_{0}(q)$. The difference between these functionals cannot be arbitrarily small. Here we derive the smallest possible value of the variation of the total deviations from its minimum, $\delta T D$, also called, the error of the total deviations value. 
First, we mention that the transition of the continuous to the discrete way for describing the values of $x$, can be realized as follows:

$$
\int_{a}^{b}(\cdots) d x=\lim _{N \rightarrow \infty} \frac{b-a}{N} \cdot \sum_{i=1}^{N}(\cdots)
$$

while the expression of the total deviations is given by

$$
T D_{q}\left(\left\{p_{k}\right\}\right)^{q}=\int_{x \in D}\left|V\left(x ;\left\{p_{k}\right\}\right)-f(x)\right|^{q} d x \approx \frac{L}{N} \cdot \sum_{i=1}^{N}\left|V\left(x_{i} ;\left\{p_{k}\right\}\right)-f\left(x_{i}\right)\right|^{q}=x_{\text {res }} \cdot \sum_{i=1}^{N}\left|V\left(x_{i} ;\left\{p_{k}\right\}\right)-f\left(x_{i}\right)\right|^{q},
$$

for large values of $N$, where $L$ is the total length of the domain $D$, and the resolution of $x$-values is $x_{r e s}=L / N$. In the discrete case, it is sufficient to express the total deviations simply by

$$
\operatorname{TD}_{q}\left(\left\{p_{k}\right\}\right)^{q}=\sum_{i=1}^{N}\left|u_{i}\right|^{q}
$$

where we set $u_{i} \equiv u\left(x_{i} ;\left\{p_{k}\right\}\right) \equiv V\left(x_{i} ;\left\{p_{k}\right\}\right)-f\left(x_{i}\right), V_{i} \equiv V\left(x_{i} ;\left\{p_{k}\right\}\right)$ and $y_{i} \equiv f\left(x_{i}\right)$.

Then, we calculate the error of the total deviations values, $\delta T D$, near the local minimum of $T D_{q}\left(\left\{p_{k}\right\}\right)^{q}$, that is, for $\left\{p_{k}\right\}=\left\{p_{k}^{*}\right\}, \forall k=1,2, \ldots, n$. Thus,

$$
\delta T D=\left.\left[\delta D_{q}\left(\left\{p_{k}\right\}\right)^{q}\right]\right|_{\left\{p_{k}\right\}=\left\{p_{k}^{*}\right\}}=\left.\left[\delta \sum_{i=1}^{N}\left|u_{i}\right|^{q}\right]\right|_{\left\{p_{k}\right\}=\left\{p_{k}^{*}\right\}} .
$$

In the case of a large number of sampling elements, we adopt the continuous description, i.e.,

$$
\sum_{i=1}^{N}\left|u_{i}\right|^{q} \approx N \cdot\left\langle|u|^{q}\right\rangle, \text { with }\left\langle|u|^{q}\right\rangle=\int_{u \in D_{u}}|u|^{q} S(u) d u,
$$

and $S(u)$ is the distribution of $u$-values in their domain $D_{u}$, that is,

$$
\left\langle|u|^{q}\right\rangle=\frac{1}{L} \cdot \int_{x \in D}\left|V\left(x ;\left\{p_{k}\right\}\right)-f(x)\right|^{q} d x,
$$

since $x$-values are equidistributed in their domain $D$. Therefore,

$$
\delta \sum_{i=1}^{N}\left|u_{i}\right|^{q} \approx \delta N \cdot\left\langle|u|^{q}\right\rangle+N \cdot \delta\left\langle|u|^{q}\right\rangle=\left\langle|u|^{q}\right\rangle+N \cdot \delta\left\langle|u|^{q}\right\rangle \approx \frac{1}{N} \sum_{i=1}^{N}\left|u_{i}\right|^{q}+N \cdot \delta\left\langle|u|^{q}\right\rangle,
$$

where the number of the sampling elements, $N$, can be varied by 1 , thus $\delta N=1$. Hence,

$$
\left.\left.\left[\delta \sum_{i=1}^{N}\left|u_{i}\right|^{q}\right]\right|_{\left\{p_{k}\right\}=\left\{p_{k}^{*}\right\}} \approx \frac{1}{N}\left[\sum_{i=1}^{N}\left|u_{i}\right|^{q}\right]\right|_{\left\{p_{k}\right\}=\left\{p_{k}^{*}\right\}}+\left.N \cdot\left[\delta\left\langle|u|^{q}\right\rangle\right]\right|_{\left\{p_{k}\right\}=\left\{p_{k}^{*}\right\}^{*}}
$$

where

$$
\left.\left[\sum_{i=1}^{N}\left|u_{i}\right|^{q}\right]\right|_{\left\{p_{k}\right\}=\left\{p_{k}^{*}\right\}}=A_{0}(q)
$$


Moreover, we show that the far right part of Equation (12) is zero. Indeed:

$$
\delta\left\langle|u|^{q}\right\rangle=\frac{1}{L} \cdot \delta \int_{x \in D}\left|V\left(x ;\left\{p_{k}\right\}\right)-f(x)\right|^{q} d x=\frac{1}{L} \cdot \sum_{k=1}^{n} A_{1, k}(q) \delta p_{k},
$$

where

$$
A_{1, k}(q)=q \cdot \int_{x \in D}\left|V\left(x ;\left\{p_{k}\right\}\right)-f(x)\right|^{q-1} \operatorname{sign}\left[V\left(x ;\left\{p_{k}\right\}\right)-f(x)\right] \cdot \frac{\partial V\left(x ;\left\{p_{k}\right\}\right)}{\partial p_{k}} d x,
$$

and thus, we obtain:

$$
\left.\left[\delta\left\langle|u|^{q}\right\rangle\right]\right|_{\left\{p_{k}\right\}=\left\{p_{k}^{*}\right\}}=\left.\frac{1}{L} \cdot \sum_{k=1}^{n} A_{1, k}(q)\right|_{\left\{p_{k}\right\}=\left\{p_{k}^{*}\right\}} \delta p_{k}^{*}=0,
$$

leading to the set of the following $n$ normal equations:

$$
0=\left.A_{1, k}(q)\right|_{\left\{p_{k}\right\}=\left\{p_{k}^{*}\right\}}=\left.q \cdot \int_{x \in D}\left|V\left(x ;\left\{p_{k}^{*}\right\}\right)-f(x)\right|^{q-1} \operatorname{sign}\left[V\left(x ;\left\{p_{k}^{*}\right\}\right)-f(x)\right] \cdot \frac{\partial V\left(x ;\left\{p_{k}\right\}\right)}{\partial p_{k}}\right|_{\left\{p_{k}\right\}=\left\{p_{k}^{*}\right\}} d x .
$$

Finally,

$$
\left.\left[\delta \sum_{i=1}^{N}\left|u_{i}\right|^{q}\right]\right|_{\left\{p_{k}\right\}=\left\{p_{k}^{*}\right\}}=\frac{1}{N} A_{0}(q)
$$

hence:

$$
\delta T D(q)=\frac{1}{N} A_{0}(q)
$$

Similarly, for the continuous way of $x$-values, we have:

$$
\begin{gathered}
T D_{q}\left(\left\{p_{k}\right\}\right)^{q}=\int_{x \in D}|u|^{q} d x \approx x_{\text {res }} \cdot \sum_{i=1}^{N}\left|u_{i}\right|^{q}, \\
\left.\left.\delta T D \approx x_{r e s} \cdot\left[\delta \sum_{i=1}^{N}\left|u_{i}\right|^{q}\right]\right|_{\left\{p_{k}\right\}=\left\{p_{k}^{*}\right\}} \approx \frac{1}{N} x_{r e s} \cdot\left[\sum_{i=1}^{N}\left|u_{i}\right|^{q}\right]\right|_{\left\{p_{k}\right\}=\left\{p_{k}^{*}\right\}}=\frac{1}{N} A_{0}(q), \\
A_{0}(q)=\left.\left.\left[\int_{x \in D}|u|^{q} d x\right]\right|_{\left\{p_{k}\right\}=\left\{p_{k}^{*}\right\}} \approx x_{r e s} \cdot\left[\sum_{i=1}^{N}\left|u_{i}\right|^{q}\right]\right|_{\left\{p_{k}\right\}=\left\{p_{k}^{*}\right\}}, \\
\delta T D(q)=\frac{1}{N} A_{0}(q) \approx \frac{x_{r e s}}{L} A_{0}(q) .
\end{gathered}
$$

The result of Equation (20d) will be used in Section 4 on the expression of the optimal errors.

\section{The Uncertainty Manifold}

We define the deviation of the total deviations functional from its minimum, $\Delta T D \equiv T D_{q}\left(\left\{p_{k}\right\}\right)^{q}-$ $T D_{q}\left(\left\{p_{k}^{*}\right\}\right)^{q}>0$, which is expressed with the quadratic form:

$$
\Delta T D\left(\left\{\delta p_{k}\right\}\right)=\sum_{k_{1}, k_{2}=1}^{n} A_{2, k_{1} k_{2}}(q) \cdot \delta p_{k_{1}} \delta p_{k_{2}}
$$

where we set $\delta p_{k} \equiv p_{k}-p_{k}{ }^{*}, \forall k=1, \ldots, n$.

Given a particular value of $\Delta T D$, each of these parameter deviations, e.g., the $k$-th component $\delta p_{k}$, has a maximum value $\delta p_{k, \max }$. This maximum value $\delta p_{k, \max }$ of each parameter deviation $\delta p_{k}$, depends 
on the value of $\Delta T D$. The smallest possible value of $\delta p_{k, \max }$ is deduced when $\Delta T D$ also reaches its smallest value. The smallest possible value of $\delta p_{k, \max }$ interprets the error $\delta p_{k}{ }^{*}$ of the optimal parameter values $p_{k}{ }^{*}, \forall k=1,2, \ldots, n$; this is achieved when the particular value $\Delta T D$ is given by the smallest possible value of a deviation from the TD's minimum, $\delta T D$. In Section 2, we showed that $\delta T D$ equals:

$$
\delta T D(q)=\frac{1}{N} \cdot A_{0}(q)
$$

There are cases, where the total deviations value is subject to an experimental, reading, or any other type of a non-statistical error; this is, in general, called the resolution value $T_{\text {res }}$. Then, the smallest possible value $\delta T D$ is meaningful only when it stays above the threshold of $T_{\text {res }}$; in other words, $\delta T D \geq T_{\text {res }}$ or, if $A_{0} / N \leq T_{\text {res }}$, then $\delta T D=T_{\text {res }}$. Hence,

$$
\delta T D(q)= \begin{cases}\frac{1}{N} \cdot A_{0}(q), & \text { if } \frac{A_{0}}{N}>T_{\text {res }}, \\ T_{\text {res }}, & \text { if } \frac{A_{0}}{N} \leq T_{\text {res }} .\end{cases}
$$

The quadratic form in Equation (21) is positive definite, and thus it defines an $n$-dimensional paraboloid (hypersurface with a local minimum) immersed into an (n+1)-dimensional space. The corresponding $n+1$ axes are given by the $n$ parameter deviations $\left\{\delta p_{k}\right\}_{k=1}^{n}$ and the deviation $\Delta T D$, describing thus, the $(n+1)$-dimensional space as

$$
\left(\delta p_{1}, \delta p_{2}, \ldots, \delta p_{n} ; \Delta T D\right) \in\left\{D_{p_{1}} \otimes D_{p_{2}} \otimes \ldots \otimes D_{p_{n}}\right\} \otimes D_{\Delta T D} \subseteq \mathfrak{R}^{n+1},
$$

where $D_{\Delta T D}=\{\Delta T D \geq \delta T D>0 \backslash \Delta T D \in \mathfrak{R}\}$ is the domain of the deviation values, $\Delta T D$.

Given a fixed value of $\triangle T D$, and that can be the value of the smallest deviation, i.e., $\delta T D=\Delta T D\left(\left\{\delta p_{k}\right\}\right)$, the set of the parameter deviations $\left\{\delta p_{k}\right\}_{k=1}^{n}$ defines a locus of an $n$-dimensional ellipsoid, rotated with respect to the axes $\left\{\delta p_{k}\right\}_{k=1}^{n}$. This $n$-dimensional ellipsoid is bounded by the $(n-1)$-dimensional locus of intersection between the $n$-dimensional paraboloid $\Delta T D=\Delta T D\left(\left\{\delta p_{k}\right\}\right)$ and the $n$-dimensional hyperplane $\triangle T D=\delta T D$.

The $n$-dimensional ellipsoid is called uncertainty manifold, denoted by $\mathrm{U}_{n}$, for short. This is a manifold with an edge, meaning thus, its boundary, denoted by $\partial \mathrm{U}_{n}$. In general, the edge of an $n$-dimensional manifold is an $(n-1)$-dimensional manifold. Here, the edge $\partial \mathrm{U}_{n}$ involves the $(n-1)$-dimensional locus of intersection between the $n$-dimensional paraboloid $\Delta T D=\Delta T D\left(\left\{\delta p_{k}\right\}\right)$ and the $n$-dimensional hyperplane $\Delta T D=\delta T D$. The $n$-dimensional cuboid, which encloses the uncertainty manifold's edge $\partial \mathrm{U}_{n}$, is also a manifold with an edge and is denoted by $\mathrm{Uc}_{n}$. Its edge is an $(n-1)$-dimensional manifold denoted by $\partial \mathrm{Uc}_{n}$.

For example, consider the case of two-parametrical approximating functions, $V\left(x ; p_{1}, p_{2}\right)$. Then, the quadratic form of Equation (21) defines the two-dimensional paraboloid $\Delta T D=\Delta T D\left(\delta p_{1}, \delta p_{2}\right)$, immersed into the three-dimensional space with Cartesian axes given by $\left(x \equiv \delta p_{1}, y \equiv \delta p_{2}, z \equiv \Delta T D\right)$. The two-dimensional ellipsoid is defined by the space bounded by the locus $\delta T D=\Delta T D\left(\delta p_{1}, \delta p_{2}\right)$, which is the intersection of the two-dimensional paraboloid $\Delta T D=\Delta T D\left(\delta p_{1}, \delta p_{2}\right)$ and the two-dimensional hyperplane $\Delta T D=\delta T D$. For visualizing this example, see Figure 1 . 

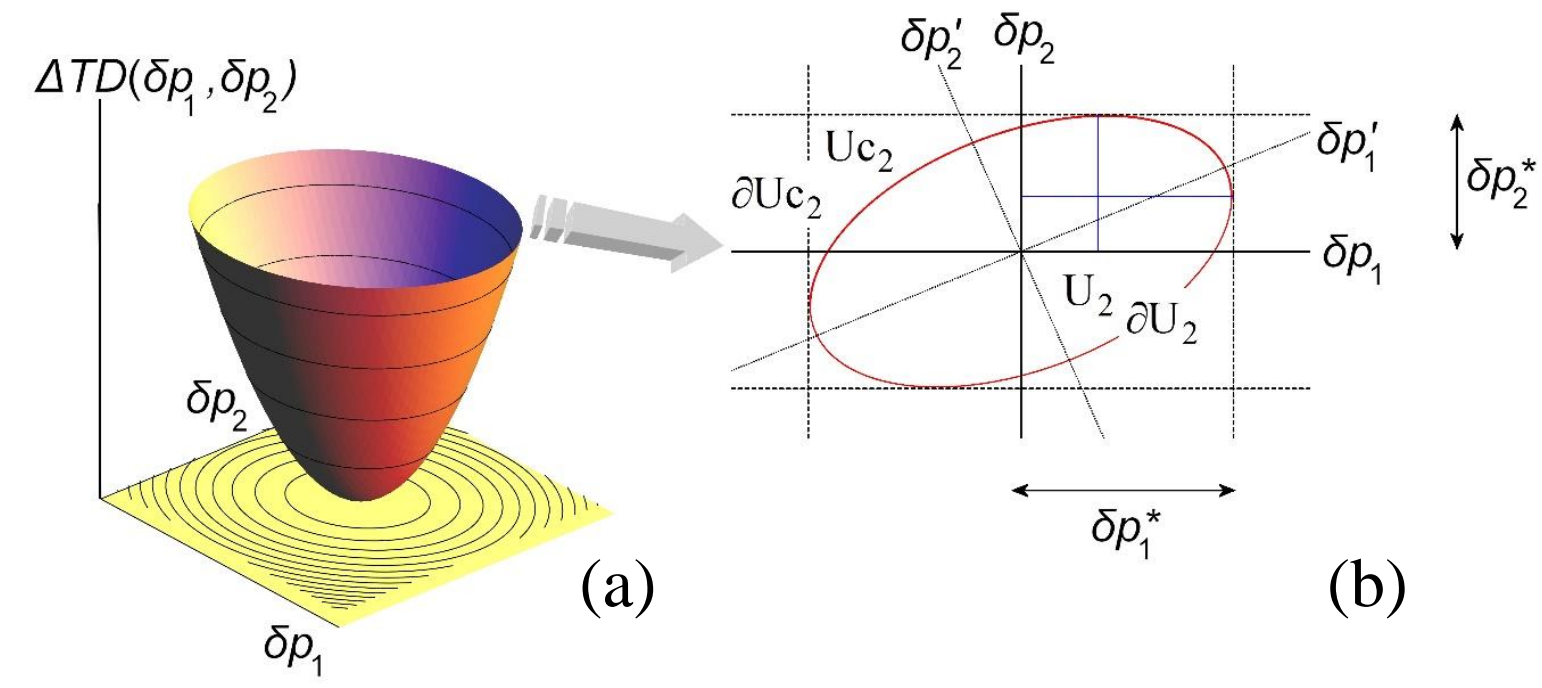

(b)

Figure 1. (a) Scheme of the $n$-dimensional paraboloid, $\Delta T D\left(\left\{\delta p_{k}\right\}\right)$, which is constructed as the deviation of the total residuals or deviations functional, total deviations (TD), from its minimum value, $A_{0}$, as shown in Equation (21); the illustrated example is for $n=2$. (b) The intersection between the paraboloid and a constant hyper-plane $\Delta T D=\delta T D$ is a rotated $n$-dimensional ellipsoid, or a rotated ellipsis for the case of $n=2$, that is the uncertainty manifold $\partial \mathrm{U}_{2}$ (enclosing $\mathrm{U}_{2}$ ). The rectangular adjusted on the extrema of $\partial \mathrm{U}_{2}$ denotes the manifold $\partial \mathrm{Uc}_{2}$ (enclosing $\mathrm{Uc}_{2}$ ).

Next, we will use the concept of the hyper-dimensional uncertainty manifold to derive the expressions of the errors of the optimal parameter values.

\section{Derivation of the Errors of the Optimal Parameter Values}

The expressions of the errors of the optimal parameter values—or simply, optimal errors-are well-known in the case of the least-square and other Euclidean based fitting methods. In [7], we have used the error expression, which is caused by the curvature, in order to have an estimate of the optimal errors (for applications, see [11-18]). Here, we will see the formal geometric derivation of the optimal error expressions.

First, we note that the edge of the uncertainty manifold, $\partial \mathrm{U}_{n}$, has a number of $n$ extrema, denoted by $\left\{C^{(k)}\right\}_{k=1}^{n}$, and they are related to the errors of the parameters optimal values, $\left\{\delta p_{k}{ }^{*}\right\}_{k=1}^{n}$, as follows:

The position vector $\vec{\Delta}^{(\mu)}$ of the corresponding point $C^{(\mu)}, \forall \mu=1,2, \ldots, n$, consists of $n$ components each, i.e., $\vec{\Delta}^{(\mu)}=\left(\Delta^{(\mu)}{ }_{1}, \Delta^{(\mu)} 2, \ldots, \Delta^{(\mu)}{ }_{n}\right)$. Thus,

$$
\begin{array}{ll}
C^{(1)}: & \left(\Delta^{(1)}{ }_{1}, \Delta^{(1)}{ }_{2}, \ldots, \Delta^{(1)} n\right)=\vec{\Delta}^{(1)} \\
C^{(2)}: & \left(\Delta^{(2)}{ }_{1} \Delta^{(2)}{ }_{2}, \ldots, \Delta^{(2)} n\right)=\vec{\Delta}^{(2)} \\
\ldots & \\
C^{(n)}: & \left(\Delta^{(n)}{ }_{1}, \Delta^{(n)}{ }_{2}, \ldots, \Delta^{(n)} n\right)=\vec{\Delta}^{(n)} .
\end{array}
$$

These components are given by the condition:

$$
\left.\left\{\frac{\partial \Delta T D\left(\left\{\delta p_{k}\right\}\right)}{\partial \delta p_{v}}\right\}\right|_{\left\{\delta p_{k}\right\}=\left\{\Delta^{(\mu)} k_{k}\right\}}=\left.\left\{\frac{\partial}{\partial \delta p_{v}} \sum_{k_{1}, k_{2}=1}^{n} A_{2, k_{1} k_{2}}(q) \cdot \delta p_{k_{1}} \cdot \delta p_{k_{2}}\right\}\right|_{\left\{\delta p_{k}\right\}=\left\{\Delta^{(\mu)} k_{k}\right\}}=0,
$$

$\forall v=1,2, \ldots, n$, with $v \neq \mu$. 
The above $(n-1)$ equations, given in Equation (26), together with

$$
\delta T D=\Delta T D\left(\left\{\delta p_{k}\right\}=\left\{\Delta_{k}^{(\mu)}\right\}\right)=\sum_{k_{1}, k_{2}=1}^{n} A_{2, k_{1} k_{2}}(q) \cdot \Delta_{k_{1}}^{(\mu)^{(\mu)}} k_{k_{2}}
$$

are sufficient for the calculation of the $n$ unknown components of $\vec{\Delta}^{(\mu)}=\left(\Delta^{(\mu)}{ }_{1}, \Delta^{(\mu)}{ }_{2}, \ldots, \Delta^{(\mu)}{ }_{n}\right)$.

The $(n-1)$ equations, given in Equation (26), arise from the fact that each of the errors of parameters optimal values, i.e., the $k$-th, $\delta p_{k}{ }^{*}$ is derived from the maximum value of the corresponding component $\delta p_{k}$, that is, $\delta p_{k, \max }$. Hence, the existence of maximum values $\left\{\delta p_{k}^{*}\right\}_{k=1}^{n}$, i.e., $\forall k=1,2, \ldots, n$, $\exists \delta p_{k}{ }^{*} \in \mathrm{U}_{n}: \forall \delta p_{k} \in \mathrm{U}_{n}, \delta p_{k}{ }^{*} \geq \delta p_{k}$, leads to the errors estimation, $\left\{\delta p_{k}{ }^{*}\right\}_{k=1}^{n}=\left\{\delta p_{k, \max }\right\}_{k=1}^{n}$. These maximum values, are located on the edge of the uncertainty manifold $\partial \mathrm{U}_{n}$, that is the hypersurface $\delta T D=\sum_{k_{1}, k_{2}=1}^{n} A_{2, k_{1} k_{2}}(q) \cdot \delta p_{k_{1}} \delta p_{k_{2}}$, i.e., $\exists \delta p_{k}{ }^{*} \in \partial \mathrm{U}_{n}$.

The maximization of the $\mu$-th parameter deviation $\delta p_{\mu}$ within uncertainty manifold is derived as follows: Since $\delta T D=\Delta T D\left(\left\{\delta p_{k}\right\}\right)$, we can express $\delta p_{\mu}$ in terms of $\delta p_{v}, \forall v=1,2, \ldots, n$ with $v \neq \mu$, i.e., $\delta p_{\mu}=\delta p_{\mu}\left(\left\{\delta p_{k}\right\}_{k=1, k \neq \mu}^{n}\right)$. Then, the procedure of finding the maximum value of $\delta p_{\mu}$, that is, $\delta p_{\mu}{ }^{*}=\left.\delta p_{\mu}\right|_{\text {max }}$ involves finding all the derivatives $\partial / \partial \delta p_{v}, \forall v=1,2, \ldots, n$ with $v \neq \mu$ of $\delta p_{\mu}=\delta p_{\mu}\left(\left\{\delta p_{k}\right\}_{k=1, k \neq \mu}^{n}\right)$, or equivalently, of $\delta T D=\Delta T D\left(\left\{\delta p_{k}\right\}\right)$ (implicit derivatives). The $(n-1)$ equations $\partial \delta p_{\mu}\left(\left\{\delta p_{k}\right\}_{k=1, k \neq \mu}^{n}\right) / \partial \delta p_{v}=0$ lead to the $(n-1)$ relationships $\delta p_{v}=\delta p_{v}\left(\delta p_{\mu}\right), \forall v=1,2, \ldots, n$ with $v \neq \mu$, which together with $\delta p_{\mu}=\delta p_{\mu}\left(\left\{\delta p_{k}\right\}_{k=1, k \neq \mu}^{n}\right)$, leads to the specific values of $\left\{\Delta^{(\mu)}{ }_{k}=\delta p_{k}\right\}_{k=1}^{n}$. Yet, only the $\mu$-th component $\Delta^{(\mu)}{ }_{\mu}=\delta p_{\mu}$ gives the error $\delta p_{\mu}{ }^{*}=\left.\delta p_{\mu}\right|_{\text {max }}$ i.e.,

$$
\delta p_{\mu}^{*}=\Delta^{(\mu)} \mu .
$$

In Appendix A, we solve Equations (26) and (27), where, we concluded that

$$
\Delta^{\left(m_{1}\right)}{ }_{m_{2}}=\frac{\sigma^{2} m_{1} m_{2}}{\sqrt{\sigma^{2} m_{1} m_{1}}}=\sqrt{\delta T D} \cdot \frac{\left(A_{2}(q)^{-1}\right)_{m_{1} m_{2}}}{\sqrt{\left(A_{2}(q)^{-1}\right)_{m_{1} m_{1}}}},
$$

or

$$
\Delta^{\left(m_{1}\right)}{ }_{m_{2}}= \begin{cases}\sqrt{\frac{1}{N} \cdot A_{0}(q)} \cdot \frac{\left(A_{2}(q)^{-1}\right)_{m_{1} m_{2}}}{\sqrt{\left(A_{2}(q)^{-1}\right)_{m_{1} m_{1}}}}, & \text { if } \frac{A_{0}}{N}>T_{\text {res }}, \\ \sqrt{T_{\text {res }}} \cdot \frac{\left(A_{2}(q)^{-1}\right)_{m_{1} m_{2}}}{\sqrt{\left(A_{2}(q)^{-1}\right)_{m_{1} m_{1}}}}, & \text { if } \frac{A_{0}}{N} \leq T_{\text {res }},\end{cases}
$$

and thus, from Equation (28), we finally derive the errors:

$$
\delta p_{k}^{*}=\Delta_{k}^{(k)}=\sqrt{\delta T D(q) \cdot\left(A_{2}(q)^{-1}\right)_{k k}}= \begin{cases}\sqrt{\frac{1}{N} \cdot A_{0}(q) \cdot\left(A_{2}(q)^{-1}\right)_{k k^{\prime}}}, & \frac{A_{0}}{N}>T_{\text {res }}, \\ \sqrt{T_{\text {res }} \cdot\left(A_{2}(q)^{-1}\right)_{k k^{\prime}}} & \frac{A_{0}}{N} \leq T_{\text {res }},\end{cases}
$$

$\forall k=1,2, \ldots, n$.

Finally, taking into account the resolution of each parameter value, $\left\{p_{r e s, k}\right\}_{k=1}^{n}$, we have:

$$
\delta p_{k}^{*}=\operatorname{Max}\left(p_{r e s, k} \sqrt{\delta T D(q) \cdot\left(A_{2}(q)^{-1}\right)_{k k}}\right),
$$

where

$$
\sqrt{\delta T D(q) \cdot\left(A_{2}(q)^{-1}\right)_{k k}}= \begin{cases}\sqrt{\frac{1}{N} \cdot A_{0}(q) \cdot\left(A_{2}(q)^{-1}\right)_{k k^{\prime}}} & \text { if } \frac{A_{0}}{N}>T_{\text {res }} \\ \sqrt{T_{\text {res }} \cdot\left(A_{2}(q)^{-1}\right)_{k k^{\prime}}} & \text { if } \frac{A_{0}}{N} \leq T_{\text {res }}\end{cases}
$$


$\forall k=1,2, \ldots, n$.

\section{Formulation of the Cases of $n=1$ and $n=2$ Dimensional Uncertainty Manifold}

\subsection{The Case of $n=1$}

Let us begin with the case of a one-dimensional paraboloid, given simply by the parabola

$$
\Delta T D(\delta p)=A_{2}(q) \cdot \delta p^{2},
$$

corresponding to uni-parametrical approximating functions. The locus of intersection between this parabola and the line $\triangle T D=\delta T D$ (that is, the one-dimensional hyperplane) are the two points $\delta p_{ \pm}= \pm \sqrt{\delta T D / A_{2}(q)}$. The uncertainty manifold $\mathrm{U}_{1}$ is the one-dimensional ellipsoid, defined by the line segment $\delta p_{-} \leq \delta p \leq \delta p_{+}$, which is enclosed by the points $\delta p_{ \pm}$. In this case, the edge of the uncertainty manifold $\partial \mathrm{U}_{1}$ is restricted to the zero-dimensional space composed only by the two points $\delta p_{ \pm}$. The manifolds $\mathrm{U}_{1}$ and $\mathrm{Uc}_{1}$ coincide (similarly with their edges, $\partial \mathrm{U}_{1}$ and $\partial \mathrm{U} c_{1}$, respectively). Hence,

$$
\delta p^{*}=\sqrt{\frac{\delta T D(q)}{A_{2}(q)}}= \begin{cases}\sqrt{\frac{1}{N} \cdot A_{0}(q) / A_{2}(q)}, & \frac{A_{0}}{N}>T_{\text {res }}, \\ \sqrt{T_{\text {res }} / A_{2}(q),} & \frac{A_{0}}{N} \leq T_{\text {res }} .\end{cases}
$$

\subsection{The Case of $n=2$}

The case of bi-parametrical approximating functions is characterized by the two-dimensional paraboloid,

$$
\Delta T D\left(\delta p_{1}, \delta p_{2}\right)=A_{11}(q) \cdot \delta p_{1}{ }^{2}+2 A_{12}(q) \cdot \delta p_{1} \delta p_{2}+A_{22}(q) \cdot \delta p_{2}{ }^{2},
$$

which is illustrated in Figure 1. The locus of intersection between this paraboloid and the plane $\Delta T D\left(\delta p_{1}, \delta p_{2}\right)=\delta T D$ is given by the rotated ellipse:

$$
\delta T D=A_{11}(q) \cdot \delta p_{1}{ }^{2}+2 A_{12}(q) \cdot \delta p_{1} \delta p_{2}+A_{22}(q) \cdot \delta p_{2}{ }^{2},
$$

written suitably as

$$
\left(\frac{\delta p^{\prime}{ }_{1}}{b_{1}}\right)^{2}+\left(\frac{\delta p_{2}^{\prime}}{b_{2}}\right)^{2}=1
$$

after the rotation transformation

$$
\begin{gathered}
\left(\begin{array}{l}
\delta p_{1} \\
\delta p_{2}
\end{array}\right)=\left(\begin{array}{cc}
\cos \theta & \sin \theta \\
-\sin \theta & \cos \theta
\end{array}\right)\left(\begin{array}{l}
\delta p^{\prime} \\
\delta p^{\prime}
\end{array}\right), \text { or } \\
\overrightarrow{\delta p}=R \overrightarrow{\delta p^{\prime}},
\end{gathered}
$$

where

$$
R=R(\theta)=\left(\begin{array}{cc}
\cos \theta & \sin \theta \\
-\sin \theta & \cos \theta
\end{array}\right), \overrightarrow{\delta p} \equiv\left(\begin{array}{c}
\delta p_{1} \\
\delta p_{2}
\end{array}\right), \overrightarrow{\delta p^{\prime}} \equiv\left(\begin{array}{c}
\delta p^{\prime}{ }_{1} \\
\delta p^{\prime}
\end{array}\right) .
$$

Then,

$$
\delta T D=\overrightarrow{\delta p}^{t} A_{2} \overrightarrow{\delta p}=\overrightarrow{\delta p^{\prime}}{ }^{t}\left(R^{t} A_{2} R\right) \overrightarrow{\delta p^{\prime}}=\overrightarrow{\delta p^{\prime}}{ }^{t} B \overrightarrow{\delta p^{\prime}},
$$

where the diagonal matrix

$$
B=B(q)=\left(\begin{array}{cc}
B_{11}(q) & 0 \\
0 & B_{22}(q)
\end{array}\right)
$$


has the following elements

$$
B_{ \pm}=\frac{1}{2}\left[A_{11}+A_{22} \pm \sqrt{\left(A_{11}-A_{22}\right)^{2}+4 A_{12}^{2}}\right], B_{11}=B_{+}, B_{22}=B_{-}
$$

which are the eigenvalues of the matrix $A_{2}(q)$. The ellipsis' major/minor axes in Equation (35) are:

$$
b_{1}=\sqrt{\frac{\delta T D}{B_{11}}}, b_{2}=\sqrt{\frac{\delta T D}{B_{22}}} .
$$

while the rotation angle $\theta$ in Equation (36) is given by

$$
\tan 2 \theta=\frac{2 A_{12}}{A_{22}-A_{11}} .
$$

The uncertainty manifold $\mathrm{U}_{2}$ is the rotated 2-dim ellipsoid in the $\left(\delta p_{1}, \delta p_{2}\right)$ axes, defined by:

$$
A_{11}(q) \cdot \delta p_{1}^{2}+2 A_{12}(q) \cdot \delta p_{1} \delta p_{2}+A_{22}(q) \cdot \delta p_{2}^{2} \leq \delta T D,
$$

or, in the rotated axes $\left(\delta p^{\prime}{ }_{1}, \delta p^{\prime}{ }_{2}\right)$, is simply given by:

$$
\left(\frac{\delta p^{\prime}}{b_{1}}\right)^{2}+\left(\frac{\delta p_{2}^{\prime}}{b_{2}}\right)^{2} \leq 1
$$

which is enclosed by the ellipse corresponding to the equal sign of Equation (45), that is the edge of the uncertainty manifold, $\partial \mathrm{U}_{2}$.

Finally, the errors are

$$
\begin{aligned}
\delta p_{1}^{*} & =\sqrt{\delta T D(q) \cdot\left(A_{2}(q)^{-1}\right)_{11}}=\sqrt{\delta T D(q) \cdot \frac{\left(A_{2}\right)_{22}(q)}{D_{A_{2}}(q)}} \\
& = \begin{cases}\sqrt{\frac{1}{N} \cdot A_{0}(q) \cdot \frac{\left(A_{2}\right)_{22}(q)}{D_{A_{2}}(q)}}, & \frac{A_{0}}{N}>T_{\text {res }}, \\
\sqrt{T_{\text {res }} \cdot \frac{\left(A_{2}\right)_{22}(q)}{D_{A_{2}}(q)}}, & \frac{A_{0}}{N} \leq T_{\text {res }},\end{cases}
\end{aligned}
$$

and

$$
\begin{aligned}
\delta p_{2}^{*} & =\sqrt{\delta T D(q) \cdot\left(A_{2}(q)^{-1}\right)_{22}}=\sqrt{\delta T D(q) \cdot \frac{\left(A_{2}\right)_{11}(q)}{D_{A_{2}}(q)}} \\
& = \begin{cases}\sqrt{\frac{1}{N} \cdot A_{0}(q) \cdot \frac{\left(A_{2}\right)_{11}(q)}{D_{A_{2}}(q)}}, & \frac{A_{0}}{N}>T_{\text {res }} \\
\sqrt{T_{\text {res }} \cdot \frac{\left(A_{2}\right)_{11}(q)}{D_{A_{2}}(q)}}, & \frac{A_{0}}{N} \leq T_{\text {res }} .\end{cases}
\end{aligned}
$$

\section{Conclusions}

The paper presented the geometric interpretation of the errors of the optimal parameter values, derived from a multi-parametrical fitting, based on a metric induced by the non-Euclidean $L_{q}$-norm. Typical fitting methods are mostly developed based on Euclidean norms, leading to the traditional least-square method. On the other hand, the theory of general fitting methods based on non-Euclidean norms, is still under development; the normal equations can provide the optimal values of the fitting parameters, while this paper completed the puzzle by improving understanding the derivations and geometric meaning of the errors.

In particular, we showed that the statistical errors of the optimal parameter values are given by the axes of the ellipsoid called uncertainty manifold, that is, the intersection of the paraboloid 
of the residuals' expansion $\Delta T D\left(\left\{\delta p_{k}\right\}\right) \equiv T D_{q}\left(\left\{p_{k}=p_{k}^{*}+\delta p_{k}\right\}\right)^{q}-T D_{q}\left(\left\{p_{k}^{*}\right\}\right)^{q}$ along the deviations $\left\{\delta p_{k}\right\}_{k=1}^{n}$, with the hyperplane $\Delta T D\left(\left\{\delta p_{k}\right\}\right)=\delta T D=$ const. The constant $\delta T D$ represents the smallest possible value of a deviation from the TD's minimum, also mentioned as an error of the value of the total deviations.

In summary, the $L_{q}$-normed fitting involves minimizing:

$$
\mathrm{TD}_{q}\left(\left\{p_{k}\right\}\right)^{q}=A_{0}(q)+\sum_{k_{1}, k_{2}=1}^{n} A_{2, k_{1} k_{2}}(q) \cdot\left(p_{k_{1}}-p_{k_{1}}{ }^{*}\right) \cdot\left(p_{k_{2}}-p_{k_{2}}{ }^{*}\right)+O\left(\left|p_{k}-p_{k}{ }^{*}\right|^{3}\right),
$$

where

$$
A_{0}(q)=\int_{x \in D}|u|^{q} d x
$$

$$
\begin{gathered}
A_{2, k_{1} k_{2}}(q)=\delta_{1 q} \cdot \gamma_{k_{1} k_{2}}+ \\
\frac{q}{2} \cdot \int_{x \in D}\left[|u|^{q-1} \cdot \operatorname{sgn}(u) \cdot \frac{\partial^{2} V\left(x ;\left\{p_{k}^{*}\right\}\right)}{\partial p_{k_{1}} \partial p_{k_{2}}}+(q-1) \cdot|u|^{q-2} \cdot \frac{\partial V\left(x ;\left\{p_{k}^{*}\right\}\right)}{\partial p_{k_{1}}} \cdot \frac{\partial V\left(x ;\left\{p_{k}^{*}\right\}\right)}{\partial p_{k_{2}}}\right] d x, \\
\gamma_{k_{1} k_{2}} \equiv \sum_{\forall i:} \frac{1}{\left|u^{\prime}\left(x_{i}\right)\right|} \cdot \frac{\partial V\left(x_{i} ;\left\{p_{k}^{*}\right\}\right)}{\partial p_{k_{1}}} \cdot \frac{\partial V\left(x_{i} ;\left\{p_{k}^{*}\right\}\right)}{\partial p_{k_{2}}} .
\end{gathered}
$$

The normal equations are given by:

$$
\int_{x \in D}|u|^{q-1} \cdot \operatorname{sgn}(u) \cdot \frac{\partial V\left(x ;\left\{p_{k}{ }^{*}\right\}\right)}{\partial p_{l}} d x=0, \forall l=1,2, \ldots, n,
$$

where we set $u=u(x) \equiv V\left(x ;\left\{p_{k}^{*}\right\}\right)-f(x)$.

Finally, we summarize the concluding relationships of the paper:

$$
\begin{gathered}
\Delta T D\left(\left\{\delta p_{k}\right\}\right) \equiv T D_{q}\left(\left\{p_{k}=p_{k}{ }^{*}+\delta p_{k}\right\}\right)^{q}-T D_{q}\left(\left\{p_{k}{ }^{*}\right\}\right)^{q}=\sum_{k_{1}, k_{2}=1}^{n} A_{2, k_{1} k_{2}}(q) \cdot \delta p_{k_{1}} \delta p_{k_{2}}, \\
\Delta T D_{\min } \equiv \operatorname{Max}\left(\delta T D(q), T_{r e s}\right), \delta T D(q)=\frac{1}{N} \cdot A_{0}(q), A_{0}(q) \equiv T D_{q}\left(\left\{p_{k}{ }^{*}\right\}\right)^{q}, \\
\delta p_{k}^{*}=\operatorname{Max}\left(p_{r e s, k}, \sqrt{\delta T D(q) \cdot\left(A_{2}(q)^{-1}\right)_{k k}}\right),
\end{gathered}
$$

with special cases:

- For $n=1$ :

$$
\delta p^{*}=\sqrt{\frac{\delta T D(q)}{A_{2}(q)}}= \begin{cases}\sqrt{\frac{1}{N} \cdot A_{0}(q) / A_{2}(q)}, & \frac{A_{0}}{N}>T_{\text {res }}, \\ \sqrt{T_{\text {res }} / A_{2}(q)}, & \frac{A_{0}}{N} \leq T_{\text {res }} .\end{cases}
$$

- $\quad$ For $n=2$ :

$$
\begin{aligned}
\delta p_{1}^{*} & =\sqrt{\delta T D(q) \cdot\left(A_{2}(q)^{-1}\right)_{11}}=\sqrt{\delta T D(q) \cdot \frac{\left(A_{2}\right)_{22}(q)}{D_{A_{2}}(q)}} \\
& = \begin{cases}\sqrt{\frac{1}{N} \cdot A_{0}(q) \cdot \frac{\left(A_{2}\right)_{22}(q)}{D_{A_{2}}(q)}}, & \frac{A_{0}}{N}>T_{\text {res }}, \\
\sqrt{T_{\text {res }} \cdot \frac{\left(A_{2}\right)_{22}(q)}{D_{A_{2}}(q)}}, & \frac{A_{0}}{N} \leq T_{\text {res }},\end{cases}
\end{aligned}
$$


and

$$
\begin{aligned}
\delta p_{2}^{*} & =\sqrt{\delta T D(q) \cdot\left(A_{2}(q)^{-1}\right)_{22}}=\sqrt{\delta T D(q) \cdot \frac{\left(A_{2}\right)_{11}(q)}{D_{A_{2}}(q)}} \\
& = \begin{cases}\sqrt{\frac{1}{N} \cdot A_{0}(q) \cdot \frac{\left(A_{2}\right)_{11}(q)}{D_{A_{2}}(q)}}, & \frac{A_{0}}{N}>T_{\text {res }}, \\
\sqrt{T_{\text {res }} \cdot \frac{\left(A_{2}\right)_{11}(q)}{D_{A_{2}}(q)}}, & \frac{A_{0}}{N} \leq T_{\text {res }} .\end{cases}
\end{aligned}
$$

Funding: This research was funded by NASA's HGI Program, grant number NNX17AB74G.

Conflicts of Interest: The author declares no conflict of interest.

\section{Appendix A. Extrema of the Uncertainty Manifold}

Here we calculate the position vectors $\left\{\vec{\Delta}^{(k)}\right\}_{k=1}^{n}$ and the maximum points $\left\{C^{(k)}\right\}_{k=1}^{n}$, used for the derivations of errors of the optimal parameter values in Section 4.

The following $(n-1)$ equations:

$$
\left.\left[\frac{\partial}{\partial \delta p_{v}} \Delta T D\left(\left\{\delta p_{k}\right\}\right)\right]\right|_{\left\{\delta p_{k}\right\}=\left\{\Delta^{(\mu)}{ }_{k}\right\}}=0,
$$

$\forall v=1,2, \ldots, n$, with $v \neq \mu$, together with the one of

$$
\delta T D=\Delta T D\left(\left\{\delta p_{k}\right\}=\left\{\Delta_{k}^{(\mu)}\right\}\right)=\sum_{k_{1}, k_{2}=1}^{n} A_{2, k_{1} k_{2}}(q) \cdot \Delta^{(\mu)} k_{1} \Delta^{(\mu)} k_{2},
$$

are sufficient for the calculation of the $n$ unknown components of $\vec{\Delta}^{(\mu)}=\left(\Delta^{(\mu)} 1, \Delta^{(\mu)}{ }_{2}, \ldots, \Delta^{(\mu)} n\right)$. Then,

$$
\begin{aligned}
& \left.\left\{\frac{\partial}{\partial \delta p_{v}}\left[\Delta T D\left(\left\{\delta p_{k}\right\}\right)\right]\right\}\right|_{\left\{\delta p_{k}\right\}=\left\{\Delta^{\left.(\mu)_{k}\right\}}\right.}=\left.\left\{\frac{\partial}{\partial \delta p_{v}}\left[\sum_{k_{1}, k_{2}=1}^{n} A_{2, k_{1} k_{2}}(q) \cdot \delta p_{k_{1}} \cdot \delta p_{k_{2}}\right]\right\}\right|_{\left.\left\{\delta p_{k}\right\}=\left\{\Delta^{(\mu)}\right)_{k}\right\}} \\
& =\left.\left\{\sum_{k_{1}, k_{2}=1}^{n} A_{2, k_{1} k_{2}}(q) \cdot \delta p_{k_{1}} \cdot \delta_{k_{2} v}+\sum_{k_{1}, k_{2}=1}^{n} A_{2, k_{1} k_{2}}(q) \cdot \delta p_{k_{2}} \cdot \delta k_{k_{1} v}\right\}\right|_{\left\{\delta p_{k}\right\}=\left\{\Delta^{(\mu)}{ }_{k}\right\}} \\
& =\left.2\left\{\sum_{k=1}^{n} A_{2, v k}(q) \cdot \delta p_{k}\right\}\right|_{\left.\left\{\delta p_{k}\right\}=\left\{\Delta^{(\mu)}\right)_{k}\right\}}=2 \cdot \sum_{k=1}^{n} A_{2, v k}(q) \cdot \Delta^{(\mu)_{k}}
\end{aligned}
$$

(where we used the Kronecker's delta, $\delta_{m n}=1$ for $m=n$ and 0 for $m \neq n$ ). Hence,

$$
\sum_{k=1}^{n} A_{2, v k}(q) \cdot \Delta_{k}^{(\mu)}=0
$$

$\forall v=1,2, \ldots, n$, with $v \neq \mu$. Setting:

$$
\sum_{k=1}^{n} A_{2, \mu k}(q) \cdot \Delta_{k}^{(\mu)} \equiv \zeta_{\mu}
$$

we have

$$
\zeta_{\mu} \cdot \hat{e}_{\mu}=\left(A_{2}\right) \vec{\Delta}^{(\mu)},
$$


where

$$
\hat{e}_{\mu} \equiv\left(\begin{array}{c}
0 \\
\vdots \\
0 \\
1 \\
0 \\
\vdots \\
0
\end{array}\right)=\left(\left\{\hat{e}_{\mu, k}=0\right\}_{k=1, k \neq \mu}^{n} ; \hat{e}_{\mu, \mu}=1\right) .
$$

Inversing Equation (A6), we obtain

$$
\vec{\Delta}^{(\mu)}=\zeta_{\mu} \cdot\left(A_{2}{ }^{-1}\right) \hat{e}_{\mu} .
$$

or

$$
\Delta^{(\mu)}{ }_{m}=\left(A_{2}^{-1}\right)_{m \mu} \cdot \zeta_{\mu},
$$

$\forall m=1,2, \ldots, n$. Then,

$$
\begin{aligned}
& \delta T D=\sum_{k_{1}, k_{2}=1}^{n} A_{2, k_{1} k_{2}} \cdot \Delta^{(\mu)_{k_{1}}} \Delta^{(\mu)_{k_{2}}}=\sum_{k_{1}, k_{2}=1}^{n} A_{2, k_{1} k_{2}} \cdot\left(A_{2}^{-1}\right)_{k_{1} \mu} \cdot \zeta_{\mu} \cdot\left(A_{2}^{-1}\right)_{k_{2} \mu} \cdot \zeta_{\mu} \\
& =\zeta_{\mu}^{2} \sum_{k_{1}, k_{2}=1}^{n} A_{2, k_{1} k_{2}} \cdot\left(A_{2}^{-1}\right)_{k_{1} \mu}\left(A_{2}^{-1}\right)_{k_{2} \mu}=\zeta_{\mu}^{2} \sum_{k_{2}=1}^{n} \delta_{k_{2} \mu} \cdot\left(A_{2}^{-1}\right)_{k_{2} \mu}=\zeta_{\mu}^{2} \cdot\left(A_{2}^{-1}\right)_{\mu \mu^{\prime}}
\end{aligned}
$$

thus,

$$
\zeta_{\mu}=\sqrt{\frac{\delta T D}{\left(A_{2}^{-1}\right)_{\mu \mu}}},
$$

or

$$
\Delta^{(\mu)}{ }_{m}=\left(A_{2}{ }^{-1}\right)_{m \mu} \cdot \sqrt{\frac{\delta T D}{\left(A_{2}^{-1}\right)_{\mu \mu}}},
$$

$\forall m=1,2, \ldots, n$, and by refreshing the indices, we end up with

$$
\Delta^{\left(m_{1}\right)}{ }_{m_{2}}=\sqrt{\delta T D} \cdot \frac{\left(A_{2}(q)^{-1}\right)_{m_{1} m_{2}}}{\sqrt{\left(A_{2}(q)^{-1}\right)_{m_{1} m_{1}}}} .
$$

\section{References}

1. Kenney, J.F.; Keeping, E.S. Linear Regression and Correlation. In Mathematics of Statistics, 3rd ed.; Part 1; Van Nostrand: Princeton, NJ, USA, 1962; pp. 252-285.

2. McCullagh, P. What is statistical model? Ann. Stat. 2002, 30, 1225-1310. [CrossRef]

3. Adèr, H.J. Modelling. In Advising on Research Methods: A Consultant's Companion; Adèr, H.J., Mellenbergh, G.J., Eds.; Johannes van Kessel Publishing: Huizen, The Netherlands, 2008; pp. 271-304.

4. Melissinos, A.C. Experiments in Modern Physics; Academic Press Inc.: London, UK, 1966; pp. 438-464.

5. Burden, R.L.; Faires, J.D. Numerical Analysis; PWS Publishing Company: New Orleans, LA, USA, 1993; pp. $437-438$.

6. Livadiotis, G.; McComas, D.J. Fitting method based on correlation maximization: Applications in Astrophysics. J. Geophys. Res. 2013, 118, 2863-2875. [CrossRef]

7. Livadiotis, G. Approach to general methods for fitting and their sensitivity. Physica A 2007, 375, 518-536. [CrossRef]

8. Livadiotis, G. Expectation values and variance based on Lp norms. Entropy 2012, 14, 2375-2396. [CrossRef] 
9. Livadiotis, G. Chi-p distribution: Characterization of the goodness of the fitting using L p norms. J. Stat. Distr. Appl. 2014, 1, 4. [CrossRef]

10. Livadiotis, G. Non-Euclidean-normed Statistical Mechanics. Physica A 2016, 445, 240-255. [CrossRef]

11. Livadiotis, G. On the convergence and law of large numbers for the non-Euclidean Lp-means. Entropy 2017, 19, 217. [CrossRef]

12. Livadiotis, G.; Moussas, $X$. The sunspot as an autonomous dynamical system: A model for the growth and decay phases of sunspots. Physica A 2007, 379, 436-458. [CrossRef]

13. Frisch, P.C.; Bzowski, M.; Livadiotis, G.; McComas, D.J.; Möbius, E.; Mueller, H.-R.; Pryor, W.R.; Schwadron, N.A.; Sokól, J.M.; Vallerga, J.V.; et al. Decades-long changes of the interstellar wind through our solar system. Science 2013, 341, 1080. [CrossRef] [PubMed]

14. Schwadron, N.A.; Mobius, E.; Kucharek, H.; Lee, M.A.; French, J.; Saul, L.; Wurz, P.; Bzowski, M.; Fuselier, S.; Livadiotis, G.; et al. Solar radiation pressure and local interstellar medium flow parameters from IBEX low energy hydrogen measurements. Astrophys. J. 2013, 775, 86. [CrossRef]

15. Livadiotis, G.; McComas, D.J. Evidence of large scale phase space quantization in plasmas. Entropy 2013, 15, 1118-1132. [CrossRef]

16. Fuselier, S.A.; Allegrini, F.; Bzowski, M.; Dayeh, M.A.; Desai, M.; Funsten, H.O.; Galli, A.; Heirtzler, D.; Janzen, P.; Kubiak, M.A.; et al. Low energy neutral atoms from the heliosheath. Astrophys. J. 2014, 784, 89. [CrossRef]

17. Zirnstein, E.J.; McComas, D.J. Using kappa functions to characterize outer heliosphere proton distributions in the presence of charge-exchange. Astrophys. J. 2015, 815, 31. [CrossRef]

18. Fuselier, S.A.; Dayeh, M.A.; Livadiodis, G.; McComas, D.J.; Ogasawara, K.; Valek, P.; Funsten, H.O. Imaging the development of the cold dense plasma sheet. Geophys. Res. Lett. 2015, 42, 7867-7873. [CrossRef]

(C) 2019 by the author. Licensee MDPI, Basel, Switzerland. This article is an open access article distributed under the terms and conditions of the Creative Commons Attribution (CC BY) license (http://creativecommons.org/licenses/by/4.0/). 